\title{
A more accurate method acquirement by a comparison of the prediction equations for estimating glomerular filtration rate in Chinese patients with obstructive nephropathy
}

Meixue Chen ${ }^{1}$, Jumei Xia', Guangchang Pei ${ }^{1}$, Ying Zhang ${ }^{1}$, Shuting Wu², Yushuang Qin², Yuanjun Deng ${ }^{1}$

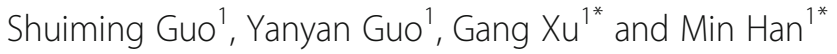

\begin{abstract}
Background: Researchers have developed several equations to predict glomerular filtration rate (GFR) in patients with chronic kidney diseases (CKD). However, there are scarcely any studies performed to discern the best equation to estimate GFR in patients with pure obstructive nephropathy. In present study, we assessed the suitability of six prediction equations and compared their performance in eGFR evaluation for Chinese patients with obstructive nephropathy.

Methods: A total of 245 adult patients with obstructive nephropathy were enrolled. We evaluated the performance of the 3 Modification of Diet in Renal Disease equations (MDRD) (the original MDRD7, 7MDRD; the abbreviated MDRD, aMDRD; and re-expressed abbreviated MDRD, re-aMDRD) and 3 Chronic Kidney Disease Epidemiology Collaboration equations (CKD-EPI) (CKD-EPI equation based on creatinine alone, CKD-EPICr; CKD-EPI equation based on cystatin C alone, CKD-EPIcys; CKD-EPI equation based on combined creatinine-cystatin, CKD-EPICr-Cys). The measured GFR (mGFR) by ${ }^{99} \mathrm{mTc}$-DTPA renal dynamic imaging method was used as the reference GFR.

Results: The mean age of the study population was $51.61 \pm 14.17$ and 131 were male (53.47\%). The mean measured GFR was $66.54 \pm 23.99 \mathrm{ml} / \mathrm{min} / 1.73 \mathrm{~m}^{2}$. Overall, the CKD-EPICr-cys equation gave the best performance with the best correlation $(R=0.72)$ and agreement $(-34.87,40.83)$. CKD-EPIcr-cys equation also exhibited the highest accuracy $(69.39 \%, P<0.01)$ and diagnostic efficacy (ROC $\left.{ }^{\text {AUC }}=0.874\right)$ with the smallest bias $(2.98, P<0.01)$. In the subgroup of the lowest GFR, CKD-EPlcys equation exhibited the highest accuracy (52.69\%) and the smallest bias (0.27). In the youngest age subgroup, CKD-EPIcys equation had the highest accuracy (71.64 \%) and the smallest bias $(-1.24)$. In other subgroups stratified by GFR, age and gender, CKD-EPIcr-cys equation remained the best performance.
\end{abstract}

Conclusion: The 3 CKD-EPI equations performed better than the 3 MDRD equations in estimating GFR in Chinese obstructive nephropathy patients; while the CKD-EPI equation based on combined creatinine-cystatin C provided the best estimation of GFR.

Keywords: Obstructive nephropathy, eGFR equation, Glomerular filtration rate

\footnotetext{
* Correspondence: xugang@tjh.tjmu.edu.cn; minhan@tjh.tjmu.edu.cn

${ }^{1}$ Division of Nephrology, Tongji Hospital, Tongji Medical College, Huazhong

University of Science and Technology, 1095 Jiefang Avenue, Wuhan, Hubei

430030, China

Full list of author information is available at the end of the article
} 


\section{Background}

Glomerular filtration rate (GFR) is an important indicator of the filtering capacity of kidneys and is considered the best overall index of renal function currently used [1]. Estimated glomerular filtration rate (eGFR) is the most important variable in the assessment of patients with suspected or known kidney disease in clinical practice [2]. Traditional methods using renal clearances of exogenous inulin, or other alternative exogenous markers (such as iothalamate, EDTA, diethylene triamine pentaacetic acid, and iohexol) can provide accurate GFR evaluation [3]. However, these tests are time consuming and expensive [4], which limits the application to monitor kidney function periodically. As an alternative, GFR estimating equations have been recommended in clinical practice. In 1976, the first creatinine clearance estimating equation - Cockcroft-Gault equation was developed [5]. From then on, researchers have developed and calibrated a series of equations to provide convenient, time-saving and reproducible estimation of kidney function, such as MDRD (Modification of Diet in Renal Disease) and CKD-EPI (Chronic Kidney Disease Epidemiology Collaboration) equations etc. [6-9]. Recently, the CKD-EPI group have developed new equations based on serum cystatin $\mathrm{C}$ and combined creatinine-cystatin
C, which were shown to perform better than the previous CKD-EPI equation based on serum creatinine alone [10]. However, the new equations have not been externally validated in a Chinese population yet. Obstructive nephropathy refers to the renal disease caused by impaired urine flow or tubular fluid [11], which is taken as one of the most common reasons for chronic kidney disease (CKD) [12-14]. It can be caused by stone, tumor, prostatic hyperplasia, etc. Long-term urinary tract obstruction can lead to renal fibrosis [15]. The pathological process of obstructive nephropathy is different from diffuse renal diseases initiated by immune mechanism, in which the damage of both kidneys are almost the same. However, obstructive nephropathy often occurs unilaterally. Even if it happens bilaterally, the degree of renal damage in the left and right kidneys is not equal. The effects of urinary tract obstruction on renal function must be considered both during and after relief of obstruction and are greatly influenced by whether the obstruction is unilateral or bilateral,acute or chronic, partial or complete [14]. To the best of our knowledge, no studies have validated these eGFR equations only in obstructive nephropathy patients. The current study aimed at testing and comparing the estimations of 6 commonly used eGFR equations (3 MDRD

Table 1 Equations to estimated GFR

\begin{tabular}{|c|c|}
\hline eGFR method & Equation \\
\hline 7MDRD & $170 \times(\mathrm{Scr})^{-0.999} \times\left(\mathrm{Age}^{\mathrm{j}-0.176} \times 0.762\right.$ (if female) $\times 1.180$ (if black) $\times(\mathrm{BUN})^{-0.170} \times(\mathrm{Alb})^{0.318}$ \\
\hline aMDRD & $186 \times(\mathrm{Scr})^{-1.154} \times(\mathrm{Age})^{-0.203} \times 0.742($ if female $) \times 1.212($ if black $)$ \\
\hline re-aMDRD & $175 \times(\mathrm{Scr})^{-1.154} \times(\text { Age })^{-0.203} \times 0.742($ if female $) \times 1.212($ if black $)$ \\
\hline \multicolumn{2}{|l|}{ CKD-EPICr } \\
\hline Female; Scr $\leq 0.7 \mathrm{mg} / \mathrm{dl}$ & $144 \times(\mathrm{Scr} / 0.7)^{-0.329} \times 0.993^{\text {Age }}[\times 1.159$ if black $]$ \\
\hline Female; Scr $>0.7 \mathrm{mg} / \mathrm{dl}$ & $144 \times(\mathrm{Scr} / 0.7)^{-1.209} \times 0.993^{\mathrm{Age}}[\times 1.159$ if black $]$ \\
\hline Male; Scr $\leq 0.9 \mathrm{mg} / \mathrm{dl}$ & $141 \times(\mathrm{Scr} / 0.9)^{-0.411} \times 0.993^{\mathrm{Age}}[\times 1.159$ if black $]$ \\
\hline Male; Scr $>0.9 \mathrm{mg} / \mathrm{dl}$ & $141 \times(\mathrm{Scr} / 0.9)^{-1.209} \times 0.993^{\mathrm{Age}}[\times 1.159$ if black $]$ \\
\hline \multicolumn{2}{|l|}{ CKD-EPIcys } \\
\hline Female or Male; Scys $\leq 0.8 \mathrm{mg} / \mathrm{dl}$ & $133 \times(\text { Scys } / 0.8)^{-0.499} \times 0.996^{\text {Age }}[\times 0.932$ if female $]$ \\
\hline Female or Male; Scys > 0.8 mg/dl & $133 \times(\text { Scys } / 0.8)^{-1.328} \times 0.996^{\text {Age }}[\times 0.932$ if female $]$ \\
\hline \multicolumn{2}{|l|}{ CKD-EPICr-cys } \\
\hline \multicolumn{2}{|l|}{ Female; } \\
\hline $\mathrm{Scr} \leq 0.7 \mathrm{mg} / \mathrm{dl} ;$ Scys $\leq 0.8 \mathrm{mg} / \mathrm{dl}$ & $130 \times(\mathrm{Scr} / 0.7)^{-0.248} \times(\mathrm{Scys} / 0.8)^{-0.375} \times 0.995^{\mathrm{Age}}[\times 1.08$ if black $]$ \\
\hline $\mathrm{Scr} \leq 0.7 \mathrm{mg} / \mathrm{dl} ;$ Scys $>0.8 \mathrm{mg} / \mathrm{dl}$ & $130 \times(\mathrm{Scr} / 0.7)^{-0.248} \times(\text { Scys } / 0.8)^{-0.711} \times 0.995^{\mathrm{Age}}[\times 1.08$ if black $]$ \\
\hline $\mathrm{Scr}>0.7 \mathrm{mg} / \mathrm{dl} ;$ Scys $\leq 0.8 \mathrm{mg} / \mathrm{dl}$ & $130 \times(\mathrm{Scr} / 0.7)^{-0.601} \times(\mathrm{Scys} / 0.8)^{-0.375} \times 0.995^{\mathrm{Age}}[\times 1.08$ if black $]$ \\
\hline $\mathrm{Scr}>0.7 \mathrm{mg} / \mathrm{dl} ;$ Scys $>0.8 \mathrm{mg} / \mathrm{dl}$ & $130 \times(\mathrm{Scr} / 0.7)^{-0.601} \times(\mathrm{Scys} / 0.8)^{-0.711} \times 0.995^{\mathrm{Age}}[\times 1.08$ if black $]$ \\
\hline \multicolumn{2}{|l|}{ Male; } \\
\hline $\mathrm{Scr} \leq 0.9 \mathrm{mg} / \mathrm{dl} ;$ Scys $\leq 0.8 \mathrm{mg} / \mathrm{dl}$ & $135 \times(\text { Scr } / 0.9)^{-0.207} \times(\text { Scys } / 0.8)^{-0.375} \times 0.995^{\text {Age }}[\times 1.08$ if black $]$ \\
\hline $\mathrm{Scr} \leq 0.9 \mathrm{mg} / \mathrm{dl} ;$ Scys $>0.8 \mathrm{mg} / \mathrm{dl}$ & $135 \times(\mathrm{Scr} / 0.9)^{-0.207} \times(\mathrm{Scys} / 0.8)^{-0.711} \times 0.995^{\mathrm{Age}}[\times 1.08$ if black $]$ \\
\hline $\mathrm{Scr}>0.9 \mathrm{mg} / \mathrm{dl} ;$ Scys $\leq 0.8 \mathrm{mg} / \mathrm{dl}$ & $135 \times(\mathrm{Scr} / 0.9)^{-0.601} \times(\mathrm{Scys} / 0.8)^{-0.375} \times 0.995^{\mathrm{Age}}[\times 1.08$ if black $]$ \\
\hline $\mathrm{Scr}>0.9 \mathrm{mg} / \mathrm{dl} ; \mathrm{Scys}>0.8 \mathrm{mg} / \mathrm{dl}$ & $135 \times(\text { Scr } / 0.9)^{-0.601} \times(\text { Scys } / 0.8)^{-0.711} \times 0.995^{\text {Age }}[\times 1.08$ if black $]$ \\
\hline
\end{tabular}


Table 2 Characteristics of the study population

\begin{tabular}{ll}
\hline Variable & Mean \pm SD $(n=245)$ or $\mathrm{n}[\%]$ \\
\hline Male gender $(\mathrm{n}[\%])$ & $131(53.5 \%)$ \\
Age (years) & $51.6 \pm 14.2$ \\
Weight $(\mathrm{kg})$ & $62.0 \pm 12.1$ \\
Height $(\mathrm{cm})$ & $163.9 \pm 7.2$ \\
BMI $\left(\mathrm{kg} / \mathrm{m}^{2}\right)$ & $23.0 \pm 3.6$ \\
BSA $\left(\mathrm{m}^{2}\right)$ & $1.7 \pm 0.2$ \\
Serum creatinine $(\mathrm{mg} / \mathrm{dl})$ & $1.2 \pm 1.1$ \\
Serum cystatin C $(\mathrm{mg} / \mathrm{l})$ & $1.4 \pm 0.7$ \\
Serum urea $(\mathrm{mg} / \mathrm{dl})$ & $18.0 \pm 10.8$ \\
Serum albumin $(\mathrm{g} / \mathrm{dl})$ & $3.7 \pm 0.5$ \\
Measured GFR $\left(\mathrm{ml} / \mathrm{min} / 1.73 \mathrm{~m}^{2}\right)$ & $67 \pm 24$ \\
\hline
\end{tabular}

BMI $\left(\mathrm{kg} / \mathrm{m}^{2}\right)=$ body weight $(\mathrm{kg}) /$ height $(\mathrm{m})^{2}$

BSA $\left(\mathrm{m}^{2}\right)=$ body weight $(\mathrm{kg})^{0.425} \times$ height $(\mathrm{cm})^{0.725} \times 0.007184$

$B M I$ body mass index, BSA body surface area, GFR glomerular filtration rate equations and 3 CKD-EPI equations), including the new developed CKD-EPI equation based on combined creatinine-cystatin $C$ [10] in pure obstructive nephropathy patients.

\section{Methods \\ Patients}

This study was performed as a retrospective study using resident patients diagnosed with obstructive nephropathy who had underwent a GFR measurement by ${ }^{99} \mathrm{mTc}$ diethylenetriamine penta-acetate $\left({ }^{99} \mathrm{mTc}-\mathrm{DTPA}\right)$ in Tongji Hospital of Huazhong University of Science and Technology (Wuhan, China) between May, 2012, and October, 2013. To be included, the age had to be at least 18 years; the biochemical results, body weight, and height had to be available from the patients' case records within 3 months of the GFR measurement. For patients who had more than one GFR measurements, the first one was used for analysis. Patients with acute kidney injury, severe edema, pleural effusion or ascites,
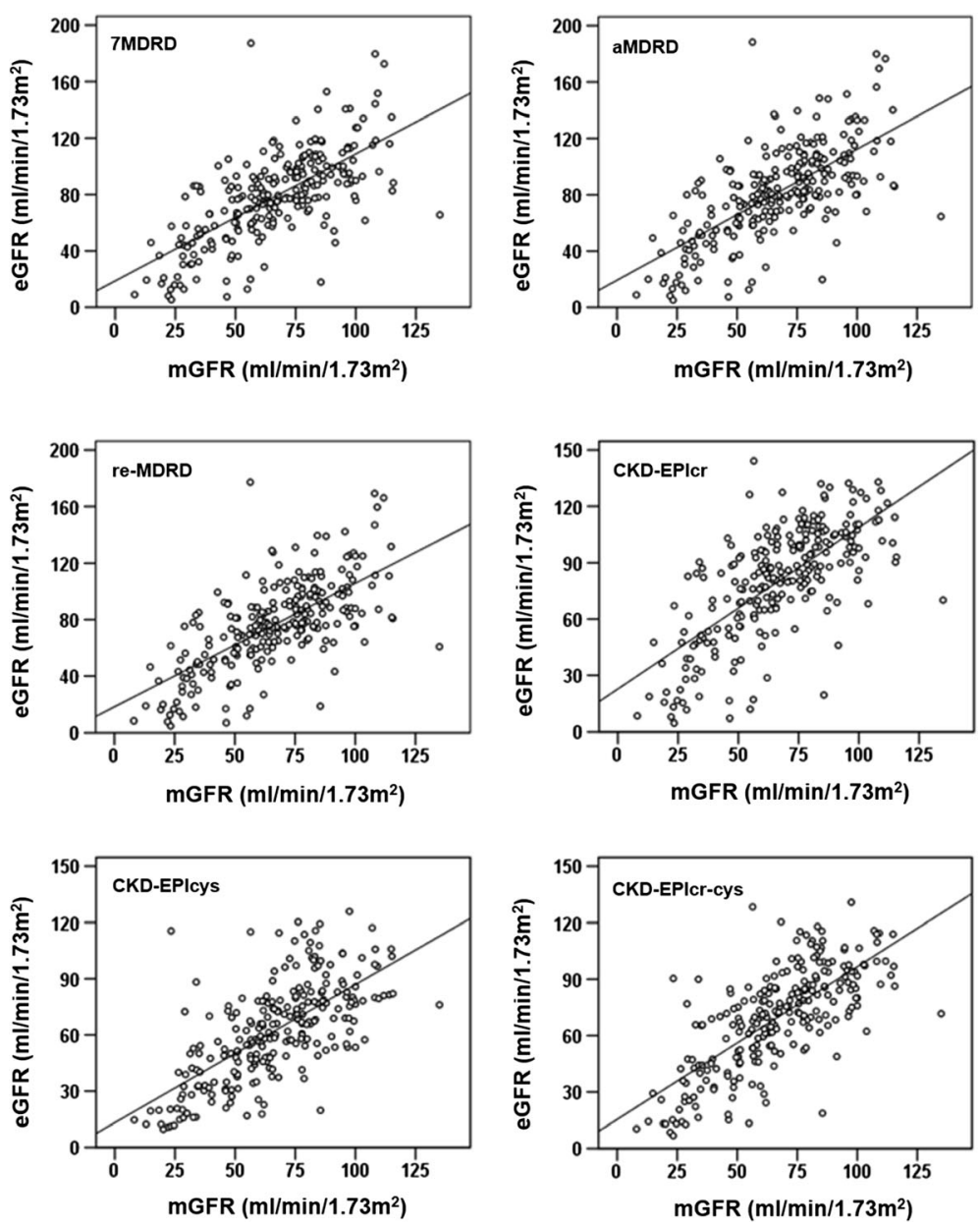

Fig. 1 Correlation between measured GFR (mGFR) and estimated GFR (eGFR) using 6 different prediction equations (univariate linear regression model was used, the solid line represents the regression line) 
malnutrition, amputation or skeletal muscle atrophy, heart failure or ketoacidosis were excluded. Patients who were taking cimetidine, trimethoprim or those who were on any kind of renal replacement therapy were also excluded [16]. Finally, a total of 245 individuals were enrolled in the present study.

\section{GFR measurements}

The measurement of GFR was performed using ${ }^{99} \mathrm{mTc}-$ DTPA renal dynamic imaging by Ifinia Hawkeye 4 SPECT (GE Healthcare, USA). The identical standard measuring method was as follows: patients were hydrated with $300 \mathrm{ml}$ of water $30 \mathrm{mins}$ before the examination. Radioactivity of the syringe containing ${ }^{99} \mathrm{mTc}$-DTPA (provided by Jiangsu Atom Medicine Research Institute, Jiangyuan
Pharmaceutical Factory) was counted before injection. And then each of the patients was given a bolus of intravenous injection of approximately $185 \mathrm{MBq}$ DTPA into the forearm. After that, the dynamic renal flow images were collected immediately using the Xeleris ${ }^{\mathrm{TM}_{3}}$ Functional Imaging Workstation (GE Healthcare Biosciences, Piscataway, NJ, USA). The post-injection syringe was also counted. Thus the difference of the syringe's radioactivity between pre- and post-injection was defined as the exact dosage of administered ${ }^{99} \mathrm{mTc}$-DTPA. The calculation of GFR values was done by the Xeleris ${ }^{\mathrm{TM}_{3}}$ Functional Imaging Workstation automatically according to the modified Gate's equation $[17,18]$. The measured GFR (mGFR) was standardized by body surface area (BSA) [15].
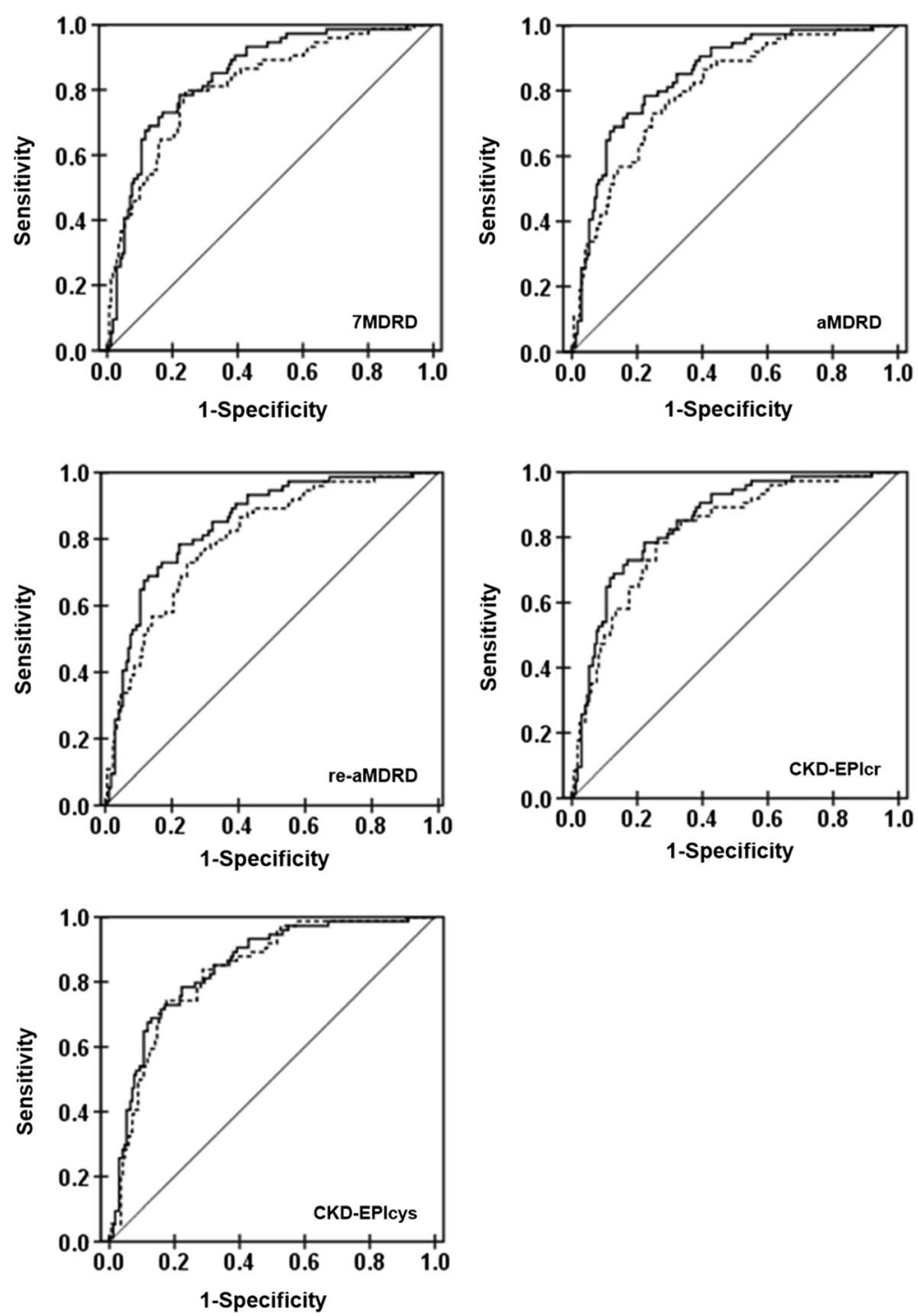

Fig. 2 Curves of Receiver Operating Characteristics analysis for the diagnostic ability of CKD-EPICr-cys equation (solid-line curve) versus other 5 equations (dotted-line curves). The values of the area under the curve were listed in Table 3 


\section{Biochemical measurements}

Serum creatinine was measured by Roche enzymatic assay (Shanghai Roche Diagnostic Products Co., Ltd, China). Cystatin $\mathrm{C}$ was determined by article-enhanced immunoturbidimetry assay (Beijing Leadman Biomedical Co., Ltd, China). All of the fasting blood samples were assayed on a Roche automatic biochemical analyser (cobas 8000 modular analyzer series, Roche Diagnostics Operations, Inc, USA). For patients who had multiple check results, the one nearest date of GFR measurement was used.

\section{Estimation of GFR}

Estimated GFR (eGFR) were calculated by 6 different equations; including 3 MDRD equations (the original MDRD7 [6], the abbreviated MDRD equation [7] and the reexpressed abbreviated MDRD [8] equation, hereafter referred to as the 7MDRD equation, aMDRD equation and re-aMDRD equation respectively); and 3 CKD-EPI equations (CKD-EPI equation based on creatinine alone [9], CKD-EPI equation based on cystatin $\mathrm{C}$ alone, and CKD-EPI equation based on combined creatininecystatin C [10], hereafter referred to as the CKDEPIcr equation, CKD-EPIcys equation, and CKDEPIcr-cys equation respectively). All the equations were all listed in Table 1.

\section{Statistical analysis}

All statistical analysis were performed in SPSS statistical software, version 19.0 for Windows (SPSS, Chicago, IL, USA). Population characteristics were tested with $t$ test, and all data were presented as means \pm SD. Body surface area (BSA) was calculated as follows [19]: $\mathrm{BSA}=\left(\right.$ bodyweight ${ }^{0.425}[\mathrm{inkg}] \times$ height $\left.^{0.725}[\mathrm{incm}]\right) \times 0.007184$. To compare the performance of the equations, Pearson correlation analysis and linear regression were applied to compare the correlation between measured GFR and estimated GFR (Pearson coefficient R was calculated) and bias, precision, and accuracy were also calculated as recommended [20, 21]. Bland-Altman plots were used to study the relation between the GFR and measurement error, we assessed the bias as well

Table 3 Diagnostic values of the equations

\begin{tabular}{llcc}
\hline Equation & $\mathrm{R}$ & $\mathrm{ROC}^{\mathrm{AUC}}$ & Sensitivity \\
\hline 7MDRD & 0.681 & 0.816 & 0.72 \\
aMDRD & 0.672 & 0.802 & 0.71 \\
re-aMDRD & 0.672 & 0.802 & 0.71 \\
CKD-EPIcr & 0.696 & 0.816 & 0.72 \\
CKD-EPlcys & 0.675 & 0.833 & 0.73 \\
CKD-EPIcr-Cys & 0.720 & 0.847 & 0.74 \\
\hline
\end{tabular}

$\mathrm{R}$ : coefficient of relationship with measured GFR

ROC $^{\mathrm{AUC}}$ : area under receiver operating characteristic curve as the limits of agreement (calculated as the bias plus or minus 1.96 times of the precision) [22]. We applied area under receiver operating characteristic (ROC ${ }^{\mathrm{AUC}}$ ) curve to describe the diagnostic efficacy of the equations. Paired $t$ test and McNemar test were respectively used to test the difference in bias and accuracy between the estimated equations. In addition, bias, precisions and accuracies were also analyzed within the stratifications of GFR, gender, and age, in order to assess the influences of these variables in subgroups. The cutoffs used for GFR stratification were $<60,60$ to 90 , and $>90 \mathrm{ml} / \mathrm{min} /$ $1.73 \mathrm{~m}^{2}$, and for age, it were $<45,45$ to 60 , and $>60$ years.

\section{Results}

Characteristics of the population

In total, 245 Chinese patients with obstructive nephropathy were included in this study, including 131 males and 114 females with a mean age of $51.61 \pm 14.17$ years. The average value of the measured GFR was $66.54 \pm 23.99 \mathrm{ml} / \mathrm{min} /$ $1.73 \mathrm{~m}^{2}$. Detailed laboratory measurements and basic characteristics of the study population were presented in Table 2 .

\section{Association and agreement between estimated GFR and measured GFR}

The overall relationships between measured and estimated GFR values were presented in Fig. 1. All of the six prediction equations correlated well with the measured GFR $(p<0.001)$ (Fig. 2). The Pearson correlation coefficients (R) varied from 0.681 to 0.720 , and CKD-EPIcr-cys equation showed the best correlation ( $R=0.720$, shown in Table 3$)$. CKD-EPIcr-cys equation also gave the highest accuracy $(P<0.01)$, the smallest bias $(P<0.01)$ (Table 4$)$ and the best diagnostic efficiency ( ROC $^{\mathrm{AUC}}=0.847, p<0.001$, Table 3 ). Furthermore, description of the agreement between estimated and measured GFR were shown in Fig. 3. CKDEPIcr-cys equation presented the best agreement with the measured GFR $(95 \%$ CI $[-34.87,40.83])$.

Table 4 Performance of the equations

\begin{tabular}{|c|c|c|c|c|c|}
\hline \multirow[t]{2}{*}{ Equation } & \multirow[t]{2}{*}{ Bias } & \multirow[t]{2}{*}{ Precision } & \multicolumn{3}{|c|}{ Accuracy(\%) } \\
\hline & & & $\leq 15 \%$ & $\leq 30 \%$ & $\leq 50 \%$ \\
\hline 7MDRD & $12.14^{* *}$ & $23.33^{* *}$ & $34.29^{* *}$ & $57.55^{* *}$ & $78.37^{* *}$ \\
\hline aMDRD & $14.77^{* *}$ & $24.65^{* *}$ & $29.80^{* *}$ & $53.47^{* *}$ & $74.69^{* *}$ \\
\hline re-aMDRD & $9.96^{* *}$ & $23.33^{* *}$ & $32.65^{* *}$ & $60.41^{* *}$ & $80.41^{* *}$ \\
\hline CKD-EPICr & $13.51^{* *}$ & $21.58^{* *}$ & $30.61^{* *}$ & $53.88^{* *}$ & $78.78^{* *}$ \\
\hline CKD-EPIcys & $-4.24^{* *}$ & $20.32^{* *}$ & $38.78^{* *}$ & 67.76 & 88.16 \\
\hline CKD-EPIcr-cys & 2.98 & 19.31 & 44.49 & 69.39 & 87.35 \\
\hline
\end{tabular}




\section{Performance of equations in subgroups}

In subgroups stratified by GFR, CKD-EPIcys equation exhibited the highest accuracy (52.69 \%) and the smallest bias (0.27) in the group of the lowest GFR, while CKDEPIcys equation and CKD-EPIcr-cys equation had an equally accuracy in patients with a GFR between 60 and $90 \mathrm{ml} / \mathrm{min} / 1.73 \mathrm{~m}^{2}$. In the group of the highest GFR, CKDEPIcr-cys equation remained the best, but both of them had larger bias in this group. For subgroups stratified by age, CKD-EPIcys equation had the best performance in the group of the youngest patients, with the highest accuracy $(71.64 \%)$ and the lowest bias $(-1.24)$; in the other 2 age groups, CKD-EPIcr-cys equation still did the best. The bias of all the 6 equations was larger in women than in men. For both genders, the highest accuracy was reached by CKD-EPIcr-cys equation. Mean bias and precision for subgroups were presented in Fig. 4, and the values were shown in Table 5 . In general, the 3
CKD-EPI equations provided better performance than the 3 MDRD equations, while CKD-EPIcr-cys equation gave the best correlation, the highest accuracy $(P<0.01)$ and diagnostic efficacy, and also the smallest bias $(P<0.01)$.

\section{Discussion}

Periodic monitoring of kidney function by a convenient and accurate method is necessary for early diagnosis of renal disease, individualized treatment and prognosis evaluation in clinical practice [3]. This pragmatic study was performed to compare 6 relatively popular GFR estimation equations in 245 Chinese patients with obstructive nephropathy, in order to test the applicability of the equations to pure obstructive nephropathy. As we expected, all these 6 eGFR equations were validated to estimate glomerular filtration function in obstructive nephropathy patients, and CKD-EPIcr-cys equation was more positively
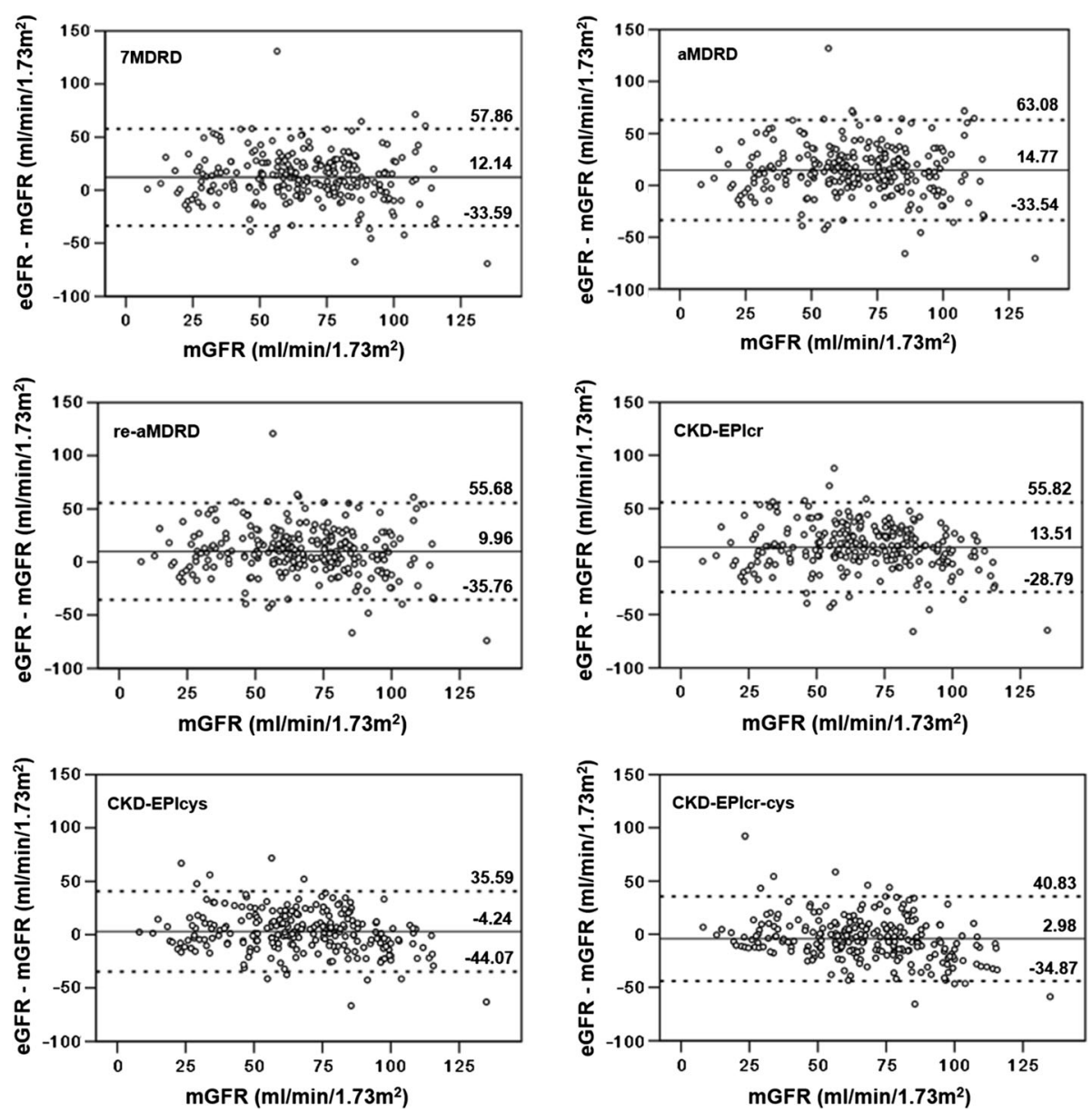

Fig. 3 Bland-Altman plots of the estimated and measured GFR. eGFR - mGFR represents the difference between the estimated GFR (eGFR) and the measured GFR (mGFR). A positive difference indicates an overestimation by the equation, whereas a negative difference indicates an underestimation. The solid lines indicate the mean difference; the dashed lines indicate the lines of agreement, calculated as the mean difference $\pm 1.96 \mathrm{SD}$ of this difference 

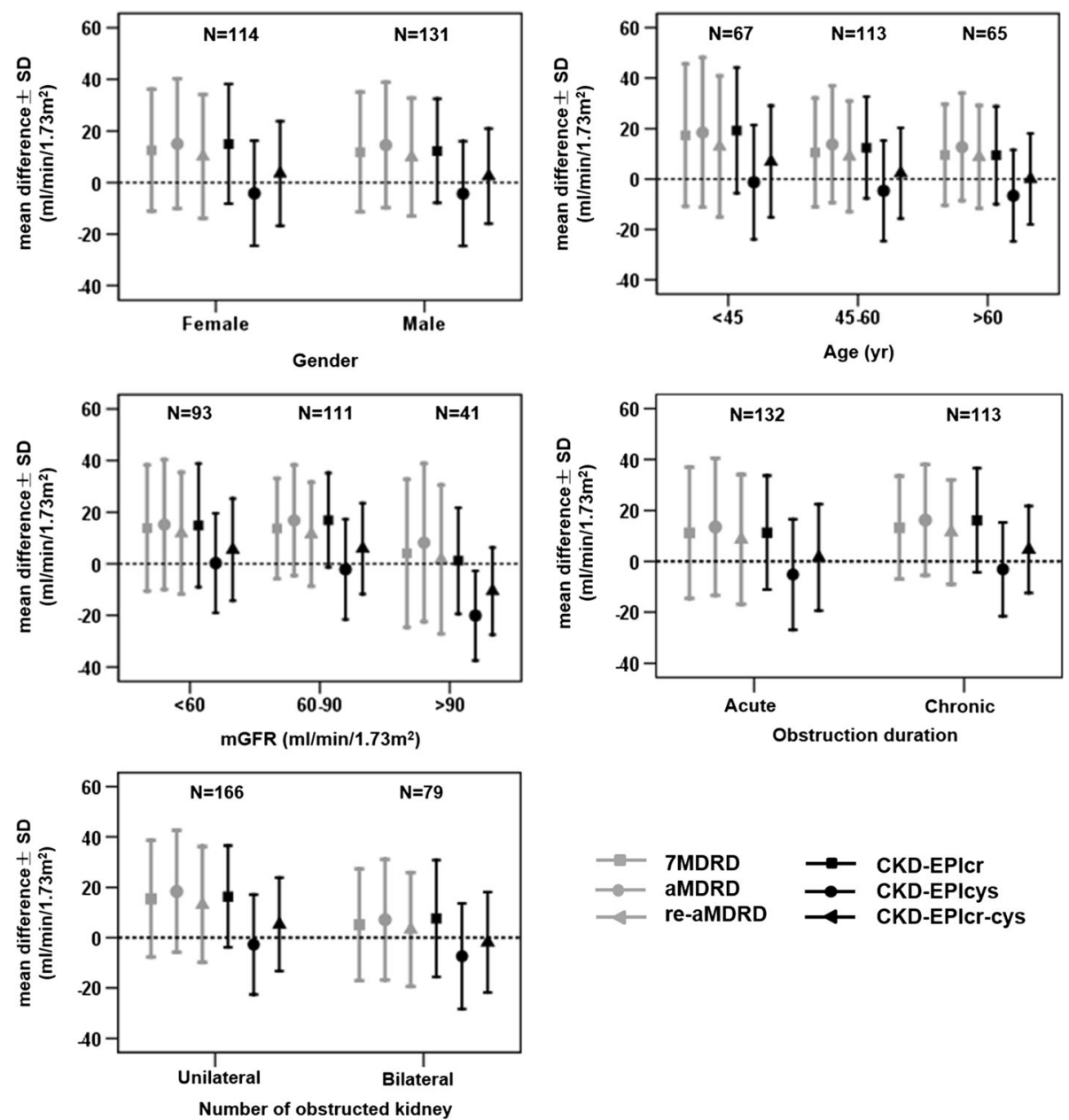

Fig. 4 Comparison of the mean bias and precision over subgroups stratified by gender, age, and measured GFR. The mean bias was calculated as the mean of the differences between the estimated and measured GFR per subgroup, whereas the precision was the SD of this difference

correlated with the ${ }^{99} \mathrm{mTc}$-DTPA GFR and had higher accuracy, even if there is the difference of pathogenesis, pathological and physiological changes and prognosis between obstructive nephropathy and diffuse renal diseases. Of all the 6 equations, the 3 CKD-EPI equations are generally more accurate than the 3 MDRD equations. Among the 3 MDRD equations, aMDRD equation is simpler to use than 7MDRD equation because it does not require inclusion of serum urea nitrogen and albumin concentration. Exclusion of these variables may also make the equation less susceptible to conditions in which serum urea nitrogen or albumin is strongly influenced by factors other than GFR. However, in our study, aMDRD equation did not perform better than 7MDRD equation. It was probably because of the difference in the serum creatinine measuring assay, which was assayed by the kinetic alkaline picrate in the original study of 7MDRD equation [6]. While in our study, it was measured by Roche enzymatic assay, which was more comparable to isotope dilution mass spectrometry (IDMS) -assigned values [8]. This may also explain the better performance of re-aMDRD equation, because re-aMDRD equation was developed by standardizing serum creatinine levels to an assay traceable to IDMS $[8,23]$. The Kidney Disease Improving Global Outcomes (KDIGO) 2012 Clinical Practice Guideline for the Evaluation and Management of Chronic Kidney Disease recommended use of the 2009 CKDEPI equation (CKD-EPICr) instead of the MDRD study equation to estimate GFR from serum creatinine. And they suggest to use the 2012 CKD-EPI equations for GFR estimation (CKD-EPIcys, CKDEPIcr-cys) [24]. It has already been validated that the 2012 CKD-EPI equations are applicable for Chinese population [25]. And our study results are also consistent with the guideline. There are several possible reasons for the best performance of the CKD-EPIcrcys equation. Firstly, compared with MDRD equations, development and validation of the 3 CKD-EPI 
Table 5 Performance of the equations in subgroups

\begin{tabular}{|c|c|c|c|c|c|c|c|c|c|c|c|c|c|c|c|c|c|c|c|}
\hline \multirow[t]{2}{*}{ Variable } & \multirow[t]{2}{*}{ Subgroup } & \multicolumn{3}{|c|}{ 7MDRD } & \multicolumn{3}{|c|}{ aMDRD } & \multicolumn{3}{|c|}{ re-aMDRD } & \multicolumn{3}{|c|}{ CKD-EPICr } & \multicolumn{3}{|c|}{ CKD-EPIcys } & \multicolumn{3}{|c|}{ CKD-EPIcr-cys } \\
\hline & & $B$ & $P$ & A(30 \%) & $\bar{B}$ & $P$ & A(30 \%) & $B$ & $P$ & $A(30 \%)$ & $B$ & $P$ & $\mathrm{~A}(30 \%)$ & $\bar{B}$ & $P$ & $A(30 \%)$ & $\bar{B}$ & $P$ & $A(30 \%)$ \\
\hline \multirow{3}{*}{$\begin{array}{l}\text { GFR } \\
\left(\mathrm{ml} / \mathrm{min} / 1.73 \mathrm{~m}^{2}\right)\end{array}$} & $<60$ & 13.83 & 24.39 & 40.86 & 15.20 & 25.13 & 39.78 & 11.82 & 23.57 & 43.01 & 14.88 & 23.90 & 37.63 & 0.27 & 19.33 & 52.69 & 5.49 & 19.83 & 51.61 \\
\hline & $60-90$ & 13.69 & 19.51 & 64.86 & 16.84 & 21.39 & 59.46 & 11.46 & 20.19 & 68.47 & 16.92 & 18.21 & 54.95 & -2.15 & 19.45 & 76.58 & 5.90 & 17.64 & 76.58 \\
\hline & $>90$ & 4.08 & 28.73 & 75.61 & 8.18 & 30.63 & 68.29 & 1.68 & 28.91 & 78.05 & 1.19 & 20.60 & 87.80 & -20.11 & 17.39 & 78.05 & -10.62 & 16.94 & 90.24 \\
\hline \multirow{3}{*}{$\begin{array}{l}\text { Age } \\
\text { (year) }\end{array}$} & $<45$ & 17.39 & 28.23 & 55.22 & 18.48 & 29.66 & 50.75 & 12.87 & 27.96 & 61.19 & 19.25 & 24.86 & 47.76 & -1.24 & 22.72 & 71.64 & 6.92 & 22.09 & 64.18 \\
\hline & $45-60$ & 10.48 & 21.54 & 59.29 & 13.76 & 23.13 & 56.64 & 8.92 & 21.91 & 64.60 & 12.44 & 20.14 & 56.64 & -4.66 & 19.96 & 72.57 & 2.31 & 18.00 & 75.22 \\
\hline & $>60$ & 9.61 & 20.04 & 12.70 & 12.70 & 21.28 & 50.77 & 8.77 & 20.39 & 52.31 & 9.45 & 19.39 & 55.38 & -6.60 & 18.16 & 55.38 & 0.07 & 18.05 & 64.62 \\
\hline \multirow[t]{2}{*}{ Gender } & Male & 11.80 & 23.19 & 64.12 & 14.53 & 24.31 & 56.49 & 9.83 & 22.85 & 64.12 & 12.24 & 20.12 & 59.45 & -4.30 & 20.33 & 67.18 & 2.49 & 18.47 & 73.28 \\
\hline & Female & 12.52 & 23.59 & 50.00 & 15.05 & 25.13 & 50.00 & 10.11 & 23.96 & 56.14 & 14.97 & 23.15 & 47.37 & -4.17 & 20.40 & 68.42 & 3.54 & 20.30 & 64.91 \\
\hline \multirow[t]{2}{*}{ Number of obstructed kidney } & Unilateral & 15.47 & 23.16 & 58.43 & 18.4 & 24.19 & 54.82 & 13.17 & 23.01 & 63.86 & 16.32 & 20.21 & 57.23 & -2.74 & 19.87 & 72.29 & 5.29 & 18.59 & 74.70 \\
\hline & Bilateral & 5.14 & 22.24 & 55.7 & 7.15 & 23.99 & 50.63 & 3.22 & 22.66 & 53.16 & 7.61 & 23.27 & 46.84 & -7.38 & 21.03 & 58.23 & -1.88 & 20.00 & 58.23 \\
\hline \multirow[t]{2}{*}{ Disease duration } & $\geq 3$ months & 11.19 & 25.75 & 60.61 & 13.52 & 26.83 & 54.55 & 8.62 & 25.48 & 65.91 & 16.13 & 20.43 & 47.79 & -3.13 & 18.47 & 73.45 & 4.65 & 17.13 & 70.80 \\
\hline & $<3$ months & 13.24 & 20.2 & 53.98 & 16.24 & 21.73 & 52.21 & 11.53 & 20.54 & 53.98 & 11.28 & 22.36 & 59.09 & -5.19 & 21.81 & 62.88 & 1.55 & 20.96 & 68.18 \\
\hline
\end{tabular}

Bias was calculated as the mean difference between estimated and measured GFR. Precision was the SD of this difference. Accuracy ( $\leq 30 \%)$ was the percentage of results deviating by $30 \%$ from the measured GFR 
equations were conducted with large databases include participants with diverse clinical characteristics, with or without kidney disease, and across a wide range of measured GFR, thus allowing more general applicability than MDRD equations $[9,10]$. Secondly, in the CKD-EPI equations, serum creatinine values are stratified according to gender and different cutoff values. However, in MDRD equations, there is no stratification. Thirdly, in comparison with MDRD, CKD-EPIcr-cys was expected to provide a more accurate estimated GFR, as cystatin C is a better glomerular filtration maker [26-28]. However, in our study, the CKD-EPI equation based on cystatin C alone was not more accurate than the creatinine estimates, suggesting that unmeasured and largely unknown non-GFR determinants of cystatin $\mathrm{C}$ are similar in magnitude to those of creatinine, which is consistent with the original publication [10]. Nevertheless, in subgroups stratified by age and gender, CKD-EPIcys equation exhibited higher accuracy than CKD-EPIcr equation. Thus, we did confirm the advantage of the cystatin C-based equation at less subject to the effects of age and gender than creatinine-based equations [8]. This study has its limitations. First, because of the retrospective data collection, most measurements of serum creatinine and GFR were not conducted on the same day. The daily changing creatinine and GFR may influence the results to some extent. Second, the measurement of serum cystatin $C$ was not calibrated to standard method as did in the original study. Third, the sample size was relatively small, the validation of the 2012 CKD-EPI equations in pure obstructive nephropathy population can not represent the whole CKD population.

\section{Conclusion}

In conclusion, the 3 CKD-EPI equations performed better than the 3 MDRD equations in estimating GFR in Chinese obstructive nephropathy patients; while among the 3 CKD-EPI equations, the CKD-EPI equation based on combined creatinine-cystatin $C$ provided the best estimation of GFR. Our data suggested that the CKD-EPI equation based on combined creatinine-cystatin $\mathrm{C}$ should be widely used in general clinical practice to assess kidney function for obstructive nephropathy patients in China.

\footnotetext{
Abbreviations

${ }^{99} \mathrm{mTc}$-DTPA: ${ }^{99} \mathrm{mTc}$-diethylenetriamine penta-acetate; CKD: Chronic kidney disease; CKD-EPI: Chronic Kidney Disease Epidemiology Collaboration; EDTA: Ethylene diamine tetraacetic acid; eGFR: estimated glomerular filtration rate; GFR: Glomerular filtration rate; IDMS: Isotope dilution mass spectrometry; KIDIGO: Kidney disease improving global outcomes; MDRD: Modification of diet in renal disease; mGFR: measured GFR; ROC $^{\text {AUC. }}$ : Receiver operating characteristic
}

\section{Acknowledgments}

Thanks to Department of Nuclear Medicine, Tongji Hospital, Tongji medical College, Huazhong University of Science and Technology for their kind help.

\section{Funding}

This work was supported by the National Natural Science Foundation of China (81370798, 81270770), National Natural Science Foundation of China for Young Scholars (81100485) and Special Projects for prevention and treatment of major diseases by the Ministry of Health of China (201002010-3) and by the Scientific Research Foundation for the Returned Overseas Chinese Scholars, State education Ministry (No.[2011]1568).

\section{Availability of supporting data}

All the data supporting our findings is contained within the manuscript.

\section{Authors' contributions}

MC, GX, MH, SW and YQ participated in the design of the study, collected data, made the figures, and analyzed and interpreted the data, drafted the manuscript. JX, GP and YZ helped to perform the statistical analysis and drafted the manuscript. YD, SG and YG participated in coordination. All authors have given final approval of the version to be published.

\section{Competing interests}

The authors declare that they have no competing interests.

\section{Ethics approval and consent to participate}

The study was carried out in accordance with the ethical standards of the Helsinki Declaration and approved by the Institution Review Board of of Huazhong University of Science and Technology, Tongji Hospital. Written informed consent was obtained from each patient before any study-specific investigation was performed.

\section{Author details}

${ }^{1}$ Division of Nephrology, Tongji Hospital, Tongji Medical College, Huazhong University of Science and Technology, 1095 Jiefang Avenue, Wuhan, Hubei 430030, China. ${ }^{2}$ Division of nuclear medicine, Tongji Hospital, Tongji Medical College, Huazhong University of Science and Technology, Wuhan 430030, China.

Received: 28 June 2015 Accepted: 5 September 2016

Published online: 18 October 2016

\section{References}

1. Ferguson MA, Waikar SS. Established and emerging markers of kidney function. Clin Chem. 2012;58:680-9.

2. Murphy DP, Hsu C. Estimating glomerular filtration rate: is it good enough? And is it time to move on? Curr Opin Nephrol Hypertens. 2013;22:310-5.

3. Stevens LA, Coresh J, Greene T, Levey AS. Assessing kidney function-measured and estimated glomerular filtration rate. N Engl J Med. 2006;354:2473-83

4. Stevens LA, Levey AS. Measurement of kidney function. Med Clin North Am. 2005:89:457-73.

5. Cockcroft DW, Gault MH. Prediction of creatinine clearance from serum creatinine. Nephron. 1976;16:31-41.

6. Levey AS, Bosch JP, Lewis J, Greene T, Rogers N, et al. A more accurate method to estimate glomerular filtration rate from serum creatinine: a new prediction equation. Ann Intern Med. 1999;130:461-70.

7. Levey AS, Greene T, Kusek JW, Beck GJ, MDRD Study Group. A simplified equation to predict glomerular filtration rate from serum creatinine [abstract]. J Am Soc Nephrol. 2000;11:155.

8. Levey AS, Coresh J, Greene T, Marsh J, Stevens LA, et al. Expressing the modification of diet in renal disease study equation for estimating glomerular filtration rate with standardized serum creatinine values. Clin Chem. 2007;53:766-72.

9. Levey AS, Stevens LA, Schmid CH, Zhang YL, Castro AF, et al. A new equation to estimate glomerular filtration rate. Ann Intern Med. 2009;150:604-12.

10. Inker $L A$, Schmid $\mathrm{CH}$, Tighiouart $\mathrm{H}$, Eckfeldt $J \mathrm{H}$, Feldman $\mathrm{HI}$, et al. Estimating glomerular filtration rate from serum creatinine and cystatin C. N Engl J Med. 2012;367:20-9. 
11. Klahr S. Obstructive nephropathy. Intern Med. 2000;39:355-61.

12. Rule AD, Bergstralh EJ, Melton LJ, Li X, Weaver AL, et al. Kidney stones and the risk for chronic kidney disease. Clin J Am Soc Nephrol. 2009;4:804-11.

13. Truong LD, Gaber L, Eknoyan G. Obstructive uropathy. Contrib Nephrol. 2011;169:311-26.

14. Wilson DR. Pathophysiology of obstructive nephropathy. Kidney Int. 1980;18:281-92.

15. Klahr S, Morrissey J. Obstructive nephropathy and renal fibrosis. Am J Physiol Renal Physiol. 2002;283:F861-75.

16. Praditpornsilpa K, Townamchai N, Chaiwatanarat T, Tiranathanagul K, Katawatin $\mathrm{P}$, et al. The need for robust validation for MDRD-based glomerular filtration rate estimation in various CKD populations. Nephrol Dial Transplant. 2011;26:2780-5.

17. Gates GF. Split renal function testing using Tc-99 m DTPA: a rapid technique for determining differential glomerular filtration. Clin Nucl Med. 1983;8:400-7.

18. Ma YC, Zuo L, Zhang CL, Wang M, Wang RF. Comparison of 99mTc-DTPA renal dynamic imaging with modified MDRD equation for glomerular filtration rate estimation in Chinese patients in different stages of chronic kidney disease. Nephrol Dial Transplant. 2007;22:417-23.

19. Du Bois $D, D u$ Bois EF. A formula to estimate the approximate surface area if height and weight be known. 1916. Nutrition. 1989;5:303.

20. Eknoyan G, Levin NW. K/DOQI clinical practice guidelines for chronic kidney disease: evaluation, classification, and stratification. Am J Kidney Dis. 2002;39:51-266.

21. Michels WM, Grootendorst DC, Verduijn M, Elliott EG, Dekker FW, et al. Performance of the Cockcroft-Gault, MDRD, and new CKD-EPI formulas in relation to GFR, age, and body size. Clin J Am Soc Nephrol. 2010;5:1003-9.

22. Martin Bland J, Altman D. Statistical methods for assessing agreement between two methods of clinical measurement. Lancet. 1986;327:307-10.

23. Levey AS, Coresh J, Greene T, Stevens LA, Zhang YL, et al. Using standardized serum creatinine values in the modification of diet in renal disease study equation for estimating glomerular filtration rate. Ann Intern Med. 2006;145:247-54.

24. Kidney Disease: Improving Global Outcomes (KDIGO) CKD Work Group. KDIGO 2012 clinical practice guideline for the evaluation and management of chronic kidney disease. Kidney Int Suppl. 2013;3:1-150.

25. Kong XL, Ma YC, Chen JH, Luo Q, Yu XQ, et al. Evaluation of the chronic kidney disease epidemiology collaboration equation for estimating glomerular filtration rate in the Chinese population. Nephrol Dial Transplant. 2013;28:641-51.

26. Tangri N, Stevens LA, Schmid CH, Zhang YL, Beck GJ, et al. Changes in dietary protein intake has no effect on serum cystatin $\mathrm{C}$ levels independent of the glomerular filtration rate. Kidney Int. 2010;79:471-7.

27. Stevens $L A$, Schmid CH, Greene T, Li L, Beck GJ, et al. Factors other than glomerular filtration rate affect serum cystatin C levels. Kidney Int 2008;75:652-60

28. Vinge $E$, Lindergård $B$, Nilsson-Ehle $P$, Grubb A. Relationships among serum cystatin $C$, serum creatinine, lean tissue mass and glomerular filtration rate in healthy adults. Scand J Clin Lab Invest. 1999;59:587-92.

\section{Submit your next manuscript to BioMed Central and we will help you at every step:}

- We accept pre-submission inquiries

- Our selector tool helps you to find the most relevant journal

- We provide round the clock customer support

- Convenient online submission

- Thorough peer review

- Inclusion in PubMed and all major indexing services

- Maximum visibility for your research

Submit your manuscript at www.biomedcentral.com/submit

Biomed Central 